\title{
Fabrication of Multimode Polymeric Waveguides and Micromirrors Using Deep X-Ray Lithography
}

\author{
Joon-Sung Kim and Jang-Joo Kim
}

\begin{abstract}
Multimode polymeric waveguides and $45^{\circ} \mathrm{mi}-$ cromirrors have been fabricated using deep X-ray lithography. Polymethylmetacrylate was used as a core layer and silica and silicone elastomer as a lower and upper cladding layer, respectively. The propagation loss of the waveguide was $0.54 \mathrm{~dB} / \mathrm{cm}$ at $830 \mathrm{~nm}$ and the loss of micromirrors was less than $0.43 \mathrm{~dB}$ at the wavelength. The $X$-ray lithography technique offers the controllability of mirror angles to $45^{\circ}$ and $-45^{\circ}$ so that it gives flexibility to the system architecture of optical interconnections.
\end{abstract}

Index Terms-Micromirror, optical interconnects, polymeric multimode waveguide, $\mathrm{X}$-ray lithography.

\section{INTRODUCTION}

W ITH RECENT advances in digital network and communication, the demand for wide bandwidth, high density, and low electromagnetic interference has been increased significantly in computing and switching systems [1], [2]. Polymeric optical waveguides are receiving much attention for planar photonic and optoelectronic application such as optical interconnects because of their relatively easy and low-cost fabrication and easy integration with other optoelectronic devices [3]-[6].

Polymeric optical multimode waveguides for optical interconnection can be fabricated by various methods such as reactive ion etching (RIE) [7], [8], molding [9], hot embossing [10], laser direct writing [5], and contact printing lithography. $45^{\circ}$ micromirrors can be fabricated using dicing with $45^{\circ}$ diamond saw [8], laser ablation [5], RIE, and molding process [10].

In this letter, we report the direct fabrication of polymethylmetacrylate (PMMA) multimode waveguides $(52 \times 50 \mu \mathrm{m})$ and micromirrors by deep X-ray lithography using synchrotron radiation. Deep X-ray lithography has been widely used in lithographie, galvanoformung, abformung (LIGA) process to fabricate high aspect ratio and large-scale microstructures such as a mold master using PMMA [11]. However, the direct fabrication of waveguides and micromirrors using the $\mathrm{X}$-ray lithography is demonstrated for the first time in this letter, to our knowledge.

$\mathrm{X}$-ray lithography is especially suitable to fabricate angled structures from the vertical direction such as micromirrors. The patterned angle by the X-ray lithography is the same as

Manuscript received January 28, 2003; revised November 19, 2003. This work was supported by the NRL, Tera-level Nanodevices Program, and the BK 21 Program. Experiments at the Pohang Light Source (PLS) were supported in part by Ministry of Science and Technology (MOST) and Pohang Steel Company (POSCO).

J.-S. Kim is with the Deptartment of Materials Science and Engineering, Kwangju Institute of Science and Technology, Gwangju 500-712, Korea.

J.-J. Kim is with the Organic Photonics Laboratory, School of Materials Science and Engineering, Seoul National University, Seoul 151-744, Korea (e-mail: jjkkim@snu.ac.kr).

Digital Object Identifier 10.1109/LPT.2004.823694

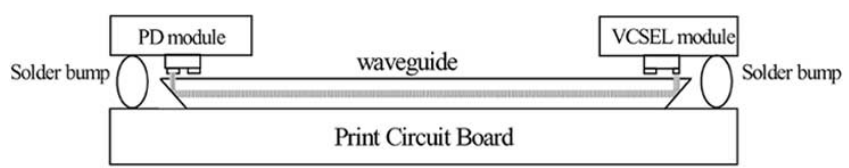

(a)
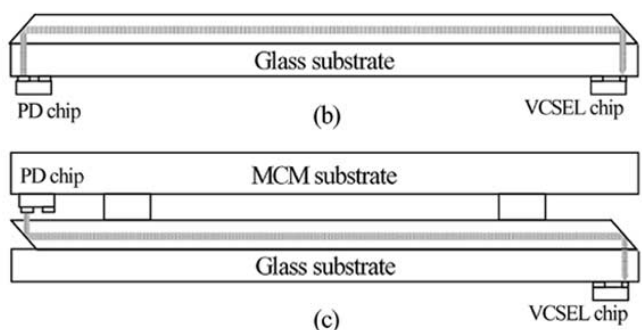

(c)

Fig. 1. Illustrations of various types of optical interconnection: VCSELs and detectors can be mounted (a) above, (b) under, and (c) opposite side of the waveguide, respectively.

the incident angle of the X-ray because of the small refraction of X-ray. Therefore, the angle of micromirrors can be easily controlled by controlling the incident angle of deep X-ray. These characteristics of X-ray lithography also allow easy fabrication of $-45^{\circ}$ (turn-up) mirrors as well as the $45^{\circ}$ (turn-down) mirrors by changing the exposure angle. It is not easy by the contact printing method because of large refraction at the air-glass-waveguide interface and the diffraction at the edge of the mask pattern during the ultraviolet (UV) exposure.

The controllability of the angle and direction of micromirrors (turn-up and turn-down) enables the fabrication of various types of optical interconnection, which is not easy by other methods. As illustrated in Fig. 1, both vertical-cavity surfaceemitting lasers (VCSELs) and detectors can be mounted above or under the waveguides, or one of them can be mounted above the waveguides while the other one is mounted below. Additionally, the sidewall roughness of the developed structure fabricated by the X-ray lithography process is less than tens of nanometers so that the extra scattering loss due to the sidewall roughness can be significantly reduced [12]. These advantages of X-ray lithography permit us to create new architectures for optical interconnection.

\section{EXPERIMENT}

Fig. 2 shows the main fabrication steps for the multimode waveguides and micromirrors using the deep X-ray lithography. A $2 \mu \mathrm{m}$-thick $\mathrm{SiO}_{2}$ layer was used as a lower cladding layer on $\mathrm{Si}$ wafer. The refractive index of $\mathrm{SiO}_{2}$ is 1.45 at $830 \mathrm{~nm}$. The surface of $\mathrm{SiO}_{2}$ was treated with a trialkoxy silane derivative to improve the adhesion of PMMA to the substrate. A 30-wt.\% 


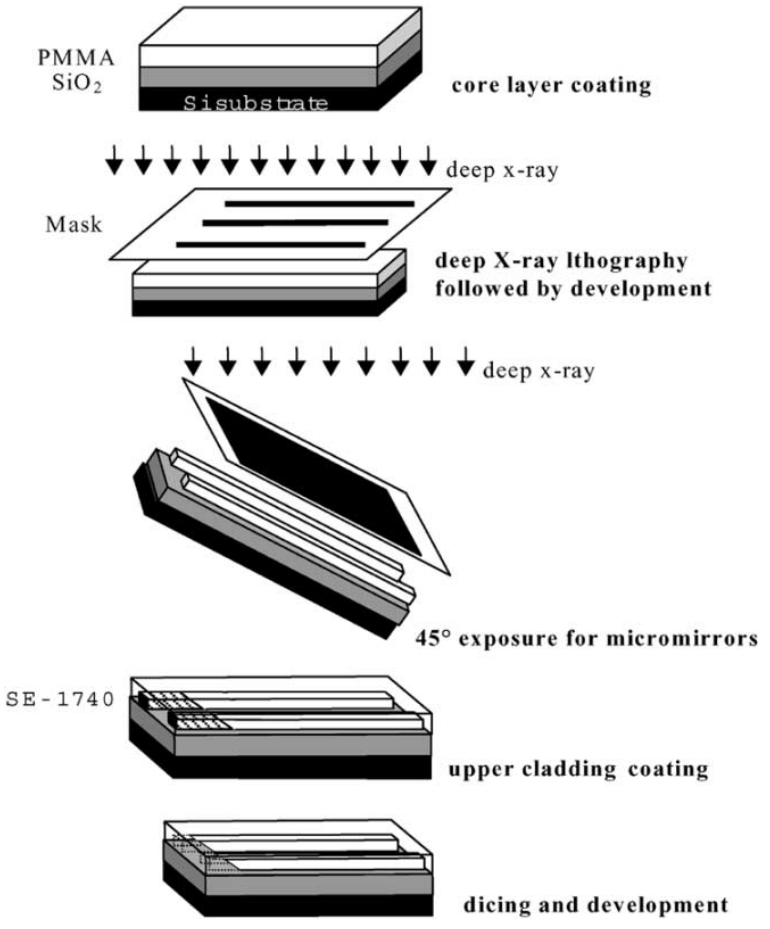

Fig. 2. Schematic diagram of fabrication steps for multimode polymeric waveguides and turn-up $45^{\circ}$ micromirrors using deep X-ray lithography.

PMMA solution in cyclohexanone was filtered with $1 \mu \mathrm{m}$ filter and spin-coated on the cladding layer. Then it was baked at $100{ }^{\circ} \mathrm{C}$ for $30 \mathrm{~min}$ followed by $160{ }^{\circ} \mathrm{C}$ for $60 \mathrm{~min}$. A PMMA film was coated twice to get a desired thickness of $52 \mu \mathrm{m}$. The refractive index of PMMA is 1.489 at the wavelength of $830 \mathrm{~nm}$. The mask for X-ray lithography was patterned with a $15-\mu \mathrm{m}$-thick Au layer on a 330- $\mu \mathrm{m}$-thick boron nitride substrate. PMMA waveguides were fabricated by the $\mathrm{X}$-ray exposure through the mask with the dose of $4 \mathrm{~kJ} / \mathrm{cm}^{3}$. The Pohang Light Source (PLS) was used as the X-ray source and the exposure time was $20 \mathrm{~min}$. After the exposure, the sample was developed in a GG developer (di-(ethylene glycol) butyl ether $60 \%$, morpholine $20 \%$, 2-aminoethanol 5\%, and DI water 15\%) at $35^{\circ} \mathrm{C}$ for $25 \mathrm{~min}$ and rinsed with DI water at $35^{\circ} \mathrm{C}$. Finally, it was dried with a gentle stream of nitrogen followed by baking on a hot plate at $100^{\circ} \mathrm{C}$ for $1 \mathrm{~h}$.

To fabricate $45^{\circ}$ and $-45^{\circ}$ micromirrors, the developed waveguides were exposed to the $\mathrm{X}$-ray radiation with an incident angle of $45^{\circ}$ and $-45^{\circ}$, respectively. After that, the exposure $80-\mu \mathrm{m}$-thick silicone elastomer (SE-1740) was spin coated on the PMMA patterns as the upper cladding layer (refractive index of 1.42) and then cured. The micromirrors in the waveguide devices were finally achieved by dicing the sample near the exposed area and developing the exposed PMMA patterns with a GG developer from diced cross section through the waveguide channel, as explained in Fig. 2.

\section{RESULTS}

The electron micrographs of PMMA multimode waveguides with a micromirror are shown in Fig. 3. The upper cladding layer was not coated in the waveguides for clarity. The multimode polymeric waveguides have a $45^{\circ}$ total reflection mirror

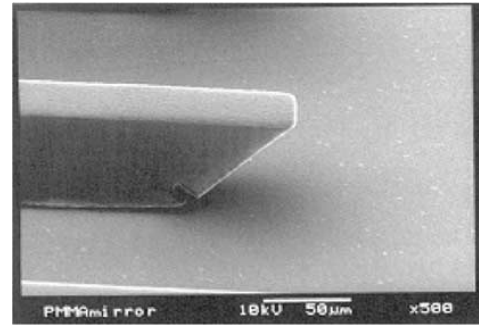

(a)

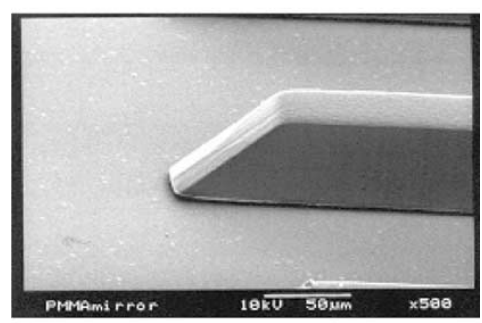

(b)

Fig. 3. Scanning electron microscope photographs of multimode polymeric waveguides with a $45^{\circ}$ total reflection mirror for (a) turn-up or (b) turn-down reflection.

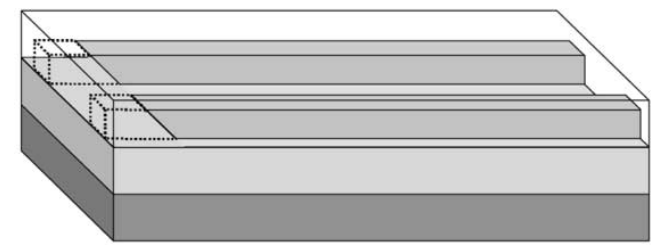

(a)

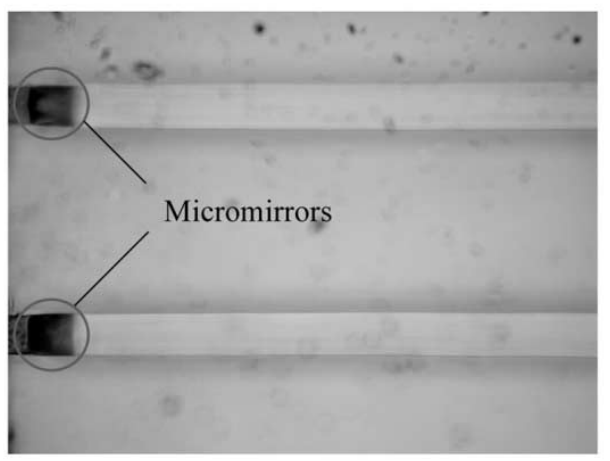

(b)

Fig. 4. (a) Schematic diagram and (b) microphotograph of fabricated multimode waveguides with the turn-up $45^{\circ}$ micromirrors.

for turn-up and turn-down reflection. The root mean square surface roughness of the PMMA waveguides measured by a surface profiler meter (Alpha-step 500) was found to be $18 \mathrm{~nm}$. Fig. 4 shows the schematic diagram and microphotograph of the fabricated waveguide devices with the turn-up $45^{\circ}$ micromirrors protected by the cladding layer from the environmental contaminations such as dusts and impurities. One should note that the micromirrors still have air-polymer interface to have high reflectance at the interface. To fabricate the structure, the exposed waveguides for the mirror fabrication was developed at the final stage after coating the upper cladding layer, as shown in Fig. 2. If the exposed waveguides had been developed be- 


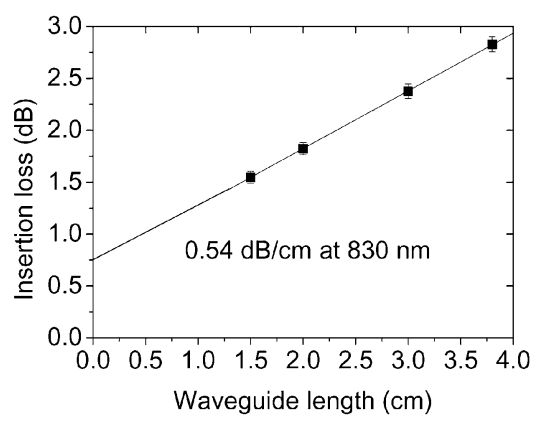

Fig. 5. Insertion losses as a function of waveguide length at the wavelength of $830 \mathrm{~nm}$.

fore spin coating the silicone elastomer, the micromirrors would have directly contacted with the material, which might result in poor reflection efficiency of the micromirrors due to the reduced refractive index difference.

The propagation loss of the multimode waveguides was measured by the cut-back method. A multimode waveguide was butt coupled to a 830-nm-wavelength laser light by a step index multimode fiber and the output light from the multimode waveguide was end-fire coupled to a photodetector (Newport 818-UV). As shown in Fig. 5, the measured propagation loss was $0.54 \mathrm{~dB} / \mathrm{cm}$ at the wavelength of $830 \mathrm{~nm}$. The coupling loss was about $0.7 \mathrm{~dB}$ for the diced end face of the waveguide.

The excess loss from the turn-up $45^{\circ}$ micromirrors was measured by comparing the insertion loss of multimode waveguides with and without micromirrors. The average excess loss due to the micromirrors was measured to be as low as $0.43 \mathrm{~dB}$ at the 830-nm wavelength. The excess loss of the micromirrors is one of the lowest values reported until now using the total internal reflection [5], [8]. The excess loss of $\sim 0.1 \mathrm{~dB}$ was reported from a metal coated mirror but with an extra process of metal coating [10]. Even though the X-ray lithography does not result in the lowest propagation and micromirror loss, combined consideration of both the propagation and micromirror loss suggests that the direct fabrication of the multimode waveguides and micromirrors using X-ray lithography has considerable potential for optical interconnection. In addition, the X-ray lithography method allows us to fabricate the various types of micromirrors which are hard to fabricate by other methods.

The waveguides are expected to be stable up to $80^{\circ} \mathrm{C}$ because the glass transition temperature of PMMA is about $100^{\circ} \mathrm{C}$ and the cladding materials have cured at an even higher temperature.

\section{CONCLUSION}

Multimode polymeric optical waveguides and $45^{\circ}$ micromirrors were fabricated by using deep X-ray lithography. Two types of $45^{\circ}$ micromirrors, i.e., $90^{\circ}$ out-of-plane optical deflections into and from their substrate, were fabricated by the control of $\mathrm{X}$-ray exposure direction. The propagation loss of the waveguides with a cross section of $52 \times 50 \mu \mathrm{m}^{2}$ was $0.54 \mathrm{~dB} / \mathrm{cm}$ at the 830-nm wavelength. The excess loss of the micromirrors was measured to be $0.43 \mathrm{~dB}$ at the wavelength. The multimode waveguides with micromirrors, fabricated using the deep X-ray lithography, have low loss and considerable potential as the new type architecture in optical interconnections.

\section{ACKNOWLEDGMENT}

The authors would like to thank S.-W. Jung (Korea Electronics Technology Institute) for fabricating a mask and for helpful discussions.

\section{REFERENCES}

[1] G. Kim, X. Han, and R. T. Chen, "An 8-Gb/s optical backplane bus based on microchannel interconnects: Design, fabrication, and performance measurements," J. Lightwave Technol., vol. 18, pp. 1477-1486, Nov. 2000

[2] G. Giaretta, W. White, M. Wegmuller, and T. Onishi, "High-speed (11 $\mathrm{Gbit} / \mathrm{s}$ ) date transmission using perfluorinated graded-index polymer optical fibers for short interconnects $(<100 \mathrm{~m})$," IEEE Photon. Technol. Lett., vol. 12, pp. 347-349, Mar. 2000.

[3] Y. S. Liu, W. B. Hennessy, R. Wojnarowsk, J. P. ristow, Y. Liu, J. Rowlette, J. Stack, J. Yardley, L. Eldada, R. M. Osgood, R. Scarmozzino, S. H. Lee, and S. Patra, "Optoelectric backplane interconnect technology development-POINT," Proc. SPIE (Optoelectronic Interconnects and Packaging IV), vol. 3005, pp. 2-10, 1997.

[4] L. Wu, B. Bihari, J. Gan, R. T. Chen, and S. Tang, "Board level optical clock signal distribution using Si-CMOS compatible polyimide-based 1-48 fanout H-tree," Proc. SPIE (Integrated Optoelectronics), vol. 3555, pp. 2-10, 1997.

[5] L. Eldada and L. W. Shacklette, "Advances in polymer integrated optics," IEEE J. Select. Topics Quantum Electron., vol. 6, pp. 54-68, Jan./Feb. 2000.

[6] T. Sakamoto, H. Tsuda, M. Hikita, T. Kagawa, K. Tateno, and C. Amano, "Optical interconnection using VCSELs and polymeric waveguide circuits," J. Lightwave Technol., vol. 18, pp. 1487-1492, Nov. 2000.

[7] J.-W. Kang, J.-P. Kim, W.-Y. Lee, J.-S. Kim, J.-S. Lee, and J.-J. Kim, "Low-loss fluorinated poly(arylene ether sulfide) waveguide with high thermal stability," J. Lightwave Technol., vol. 19, pp. 872-875, June 2001.

[8] R. Yoshimura, M. Hikita, S. Tomaru, and S. Imamura, "Low-loss polymeric optical waveguides with $45^{\circ}$ mirrors," Jpn. J. Appl. Phys., vol. 37, pp. 3657-3661, 1998.

[9] E. Voges and D. Fischer, "Multimode polymeric waveguide devices fabricated by two-component injection moulding," Electron. Lett., vol. 33, pp. 1626-1627, 1997.

[10] S. Lehmacher and A. Neyer, "Integration of polymer optical waveguides into printed circuit boards," Electron. Lett., vol. 36, pp. 1052-1053, 2000.

[11] W. Ehrfeld and A. Schmidt, "Recent developments in deep X-ray lithography," J. Vac. Sci. Technol. B, vol. 16, pp. 3526-3534, 1998.

[12] K. C. Malek and S. Yahamanyam, "Evaluation of alternative development progress for high-aspect-ratio poly(methylmethacrylate) microstructures in deep X-ray lithography," J. Vac. Sci. Technol. B, vol. 18, pp. 3354-3359, 1998. 